\title{
"Titán" y "Regio", variedades de pasto Buffel (Pennisetum ciliare) (L.) Link para zonas áridas y semiáridas
}

\section{"Titan" and "Regio", new buffelgrass varieties (Pennisetum ciliare) (L.) Link for arid and semiarid lands}

\author{
Sergio Beltrán López ${ }^{a *}$, Carlos Alberto García Díaz ${ }^{a}$, Catarina Loredo Ostib, Jorge Urrutia Morales ${ }^{a}$, \\ José Antonio Hernández Alatorrea ${ }^{a}$ Héctor Guillermo Gámez Vázqueza
}

\section{RESUMEN}

El pasto buffel (Pennisetum ciliare) es una gramínea perenne, originaria de Sud África, de buen valor forrajero que se adapta bien a una amplia gama de suelos y condiciones climáticas. Las variedades de pasto buffel Titán y buffel Regio provienen de colectas realizadas en el centro y norte de México. Fueron evaluadas en zonas áridas y semiáridas, en temporal y riego desde 1986 hasta la obtención del registro en el año 2008. Los registros definitivos otorgados por parte del Sistema Nacional de Inspección y Certificación de Semillas SNICS, son: CEN-001-060608 para buffel Titán y CEN-002-060608 para buffel Regio, siendo éstas, las primeras variedades registradas para esta especie en México. El rendimiento medio de estas variedades oscila entre 2,120 y 2,582 kg MS ha-1 $\mathrm{año}^{-1}$ en condiciones de temporal y entre 5,180 y 9,160 kg MS ha-1 año-1 en condiciones de riego. Estas variedades de pasto son tolerantes a la sequía, de fácil establecimiento y de rápido crecimiento, además, son apetecibles para el ganado, resistentes al pastoreo y tolerantes a salinidad. Actualmente, es la especie más utilizada en la rehabilitación y conservación de suelo en pastizales de zonas áridas y semiáridas en México.

PALABRAS CLAVE: Nuevas variedades, Buffel, Zonas áridas y semiáridas.

\begin{abstract}
Buffel grass ( Pennisetum ciliare) is a perennial, native grass to South Africa, from good forage value that adapts well to a wide range of soils and climatic conditions. Titan and Regio buffel grass varieties come from collections made in the Central and Northern of Mexico. They were evaluate in arid and semi-arid areas in rainfed and irrigation from 1986 until the record in 2008. The definitive records granted by the National system of inspection and certification of seeds (SNICS), are CEN-001-060608 for Titan and CEN-002-060608 for Regio buffel grass, being these the first varieties registered for this species in Mexico. The average of these varieties yield varies between 2,120 and 2,582 $\mathrm{kg} \mathrm{MS} \mathrm{ha-1}^{-1} \mathrm{yr}^{-1}$ under rainfed conditions and 5,180 to 9,160 kg MS ha-1 $\mathrm{yr}^{-1}$ under irrigation conditions. These varieties of grass are tolerant to drought, easy settlement and rapid growth; they are also appealing for livestock, grazing-resistant and tolerant to salinity. Currently, is the specie most used in the rehabilitation and conservation of soil in grassland of arid and semi-arid zones in Mexico.
\end{abstract}

KEY WORDS: New varieties, Buffelgrass, Arid and semiarid lands.

Recibido el 13 de abril de 2016. Aceptado el 17 de agosto de 2016.

Registros SNICS: Buffel Titán: CEN-001-060608; Buffel Regio: CEN-002-060608; Fecha de registro: $1^{\circ}$ de agosto de 2008.

a Investigador del INIFAP. Campo Experimental San Luis. CIRNE. México.

b Facultad de Agronomía y Veterinaria, Universidad Autónoma de San Luis Potosí. México.

*Autor de correspondencia: belseragro@gmail.com. 


\section{ORIGEN}

El pasto buffel, Pennisetum ciliare (L) Link, es una gramínea forrajera introducida a México desde los años 50s, es originaria de las zonas tropicales y subtropicales de África, India e Indonesia ${ }^{(1,2)}$. Se estima que en México existe una superficie de dos millones de hectáreas establecidas con esta especie, principalmente en los estados de Sonora, Tamaulipas, Nuevo León, Coahuila, Sinaloa y Yucatán ${ }^{(3,4)}$. El buffel es una gramínea perenne de buen valor forrajero que se adapta bien a una amplia gama de suelos y condiciones climáticas, es considerada como una de las especies más importantes introducidas en México por ser una especie tolerante a sequía, de fácil establecimiento y rápido crecimiento ${ }^{(3,5)}$, además, es apetecible para el ganado, resistente al pastoreo y tolerante a salinidad; se desarrolla bien en suelos francos y franco arenosos, donde la precipitación sea igual o mayor a $250 \mathrm{~mm}$ anuales con distribución regular durante el verano ${ }^{(6,7)}$, prospera bien desde el nivel del mar hasta los $1,800 \mathrm{~m}$ y donde la temperatura media anual es de $16{ }^{\circ} C^{(5)}$. Una de las cualidades de pasto buffel es su alto potencial de rendimiento ${ }^{(3,8,9)}$ ya que produce entre dos y diez veces más forraje que los pastos nativos ${ }^{(3,10)}$. Actualmente, es la especie más utilizada en la rehabilitación y conservación de suelo en pastizales de zonas áridas y semiáridas $^{(2)}$ señalándose inclusive, que es la especie clave para el desarrollo de la ganadería del norte y centro de México ${ }^{(3,10,11)}$.

El pasto buffel es quizá, una de las especies más estudiadas en los últimos años; los reportes incluyen diferentes tópicos, desde estudios genéticos ${ }^{(12,13)}$, fisiológicos ${ }^{(14,15)}$, ecológicos ${ }^{(9,16)}$, de establecimiento, adaptación distribución ${ }^{(3,17)}$, valor nutricional ${ }^{(18)}$ y productividad $^{(3,8)}$. Actualmente se cuenta con líneas experimentales e híbridos que se han desarrollado en México ${ }^{(18,19,20)}$ y se han utilizado variedades registradas en el extranjero(3,21,22), pero que no han sido registradas en el país, ante el Servicio Nacional de Inspección y Certificación de Semillas (SNICS), órgano oficial de la SAGARPA en México para el registro de nuevas variedades.

Las variedades de pasto buffel Titán y buffel Regio provienen de colectas realizadas en el centro y norte de México, que iniciaron en 1979 con el proyecto "Observación preliminar de especies y variedades de germoplasma forrajero". En 1982 se completó la colección de pastos siendo 78 accesiones de Cenchrus ciliaris (ahora Pennisetum ciliare). La variedad Titán (accesión 42) se colectó en la colonia Ignacio Zaragoza, municipio de Rio grande, Zacatecas, en donde se registra una altitud de $1,883 \mathrm{~m}$ y la variedad Regio (accesión 40) se colectó en el fuerte, municipio de Villanueva, Zacatecas, con una altitud de 2,170 m.

En 1986 se inició la evaluación de estas dos accesiones con el proyecto: "Evaluación de gramíneas nativas e introducidas para forraje en el Altiplano Potosino". Como resultado de estas evaluaciones, se detectaron por selección masal las accesiones 40 y 42 como sobresalientes debido a su persistencia, rendimiento y estabilidad(23); posteriormente, se evaluaron durante ocho años a partir de 1989 en diferentes ambientes, dentro del altiplano Potosino, específicamente en los municipios de Villa de Arriaga, Soledad de Graciano Sánchez y Venado S.L.P., con altitudes de 2,198, 1,835 y $1,851 \mathrm{~m}$, respectivamente.

De 2004 a 2008 se establecieron diez parcelas de validación en distintas localidades de la región árida y semiárida de San Luis Potosí para comprobar su adaptación y potencial forrajero real. Los sitios fueron: Predio El Chilar, Villa de Zaragoza (21 ${ }^{\circ} 58^{\prime} 56^{\prime \prime}$ $\mathrm{N}, 100^{\circ} 45^{\prime} 30^{\prime \prime}$ W y $\left.1,950 \mathrm{msnm}\right)$, El Pedregal, Villa de Zaragoza (215' $54^{\prime \prime}$ N, 10049'01" W y 1,860 msnm), La Sabanilla, Ejido Santa María del Refugio, Real de Catorce $\left(23^{\circ} 44^{\prime} 41^{\prime \prime} \mathrm{N}, 101^{\circ} 17^{\prime} 19^{\prime \prime}\right.$ W y 2,050 msnm), Tanque Dolores, Real de Catorce (2339'35" N, $101^{\circ} 09^{\prime} 48^{\prime \prime}$ W y 1,900 msnm), La Mora, Fracción de Triana, Salinas de Hidalgo $\left(22^{\circ} 43^{\prime} 21^{\prime \prime} \mathrm{N}\right.$, $101^{\circ} 39^{\prime} 21^{\prime \prime}$ W y 2,050 msnm), Ejido San José de la

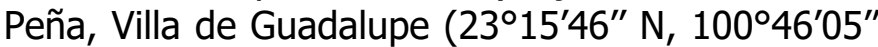
W y $1,740 \mathrm{msnm})$, La Nopalera, El Leoncito, Villa de Guadalupe $\left(23^{\circ} 22^{\prime} 50^{\prime \prime} \mathrm{N}, 100^{\circ} 45^{\prime} 18^{\prime \prime} \mathrm{W}\right.$ y 1,650 msnm), San José del Muerto, Ejido Francisco Sarabia, Matehuala $\left(23^{\circ} 22^{\prime} 00^{\prime \prime} \mathrm{N}, 100^{\circ} 48^{\prime} 33^{\prime \prime} \mathrm{W}\right.$ y 1,720 msnm), La Loma, Ejido Presa Verde, Cedral (235'ㅇ' $\mathrm{N}, 100^{\circ} 41^{\prime} 56^{\prime \prime} \mathrm{W}$ y $\left.1,910 \mathrm{msnm}\right)$ y El

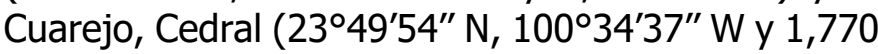
msnm).

A partir del año 2000, se inició en el Campo Experimental San Luis de INIFAP, el establecimiento 
de estas dos accesiones en un lote de $1,500 \mathrm{~m}^{2}$ cada una, con el objetivo de obtener semilla para llegar a formar dos nuevas variedades de pastos para condiciones áridas y semiáridas. En el año 2001 se inició el proyecto: "Caracterización, descripción, producción y registro de nuevas variedades de pastos", con énfasis en las accesiones sobresalientes para altitudes mayores a $1,800 \mathrm{~m}$. Con este proyecto se establecieron las dos accesiones en referencia en una superficie de 5,000 $\mathrm{m}^{2}$ cada una, con el fin de caracterizarlas y obtener semilla básica. De 2002 al 2004 se caracterizaron las variedades, se evaluó el volumen de producción de semilla y su calidad biológica (porcentajes de germinación, pureza y viabilidad).

Una vez caracterizadas, se inició el trámite de registro ante SAGARPA-SNICS para el posible registro como nuevas variedades de pasto buffel para zonas áridas y semiáridas. En el año 2008 se obtuvieron los registros definitivos por parte del SNICS: CEN-001-060608 para buffel Titán y CEN002-060608 para buffel Regio, siendo éstas, las primeras variedades registradas para esta especie en México(24).

\section{DESCRIPCIÓN MORFOLÓGICA}

\section{Características de la variedad Titán}

La raíz presenta un crecimiento fibroso con ramificaciones poco profundas (30 a $40 \mathrm{~cm}$ ); las hojas son color verde esmeralda, con forma acicular, con tamaño promedio de $29.78 \mathrm{~cm}$ de largo y 0.50 $\mathrm{cm}$ de ancho. La longevidad de las hojas es de \pm 57 días. El hábito de crecimiento de los tallos es siempre erecto, de color verde esmeralda y forma cilíndrica; el grosor medio de tallos es de $0.24 \mathrm{~cm}$ con $78.7 \mathrm{~cm}$ de longitud. Tamaño de la envoltura de la semilla $4.0 \mathrm{~mm}$, color de la envoltura de la semilla beige oscuro; altura de planta hasta la base de la inflorescencia $69.2 \mathrm{~cm}$; forma del cariópside ovoide, color café oscuro, peso $0.901 \mathrm{mg}$, largo $1.73 \mathrm{~mm}$ y ancho de $0.96 \mathrm{~mm}$; inicio de imbibición, 2.0 h (0.08 días); imbibición completa, 7.0 h (0.29 días); emergencia de radícula, 9.0 h (0.38 días); emergencia del coleoptilo $21.0 \mathrm{~h}$ (0.88 días); velocidad de germinación, $3.4 \mathrm{pl} / \mathrm{h}$; días a emergencia, 8.0; vigor de la plántula, fuerte; capacidad de establecimiento, alto; mecanismo de rebrote, con yemas basales o de la corona radical y yemas axilares; grado de amacollamiento, excelente; vigor de la recuperación, excelente; días a emisión de flores, 68; tipo de floración, indeterminado; días al inicio de antesis, nueve; días a término de floración, indeterminado; número de semillas viables por inflorescencia, 24.

\section{Características de la variedad Regio}

Raíz con ramificaciones poco profundas (40 a $50 \mathrm{~cm}$ ), y crecimiento fibroso; hojas de $16.4 \mathrm{~cm}$ de largo y $0.74 \mathrm{~cm}$ de ancho, color verde esmeralda, forma acicular; longevidad de hojas, 67 días; habito de crecimiento, erecto; tallos, color verde esmeralda, forma cilíndrica con grosor de $0.20 \mathrm{~cm}$ y longitud de $88.8 \mathrm{~cm}$; tamaño de la envoltura de la semilla, $4.0 \mathrm{~mm}$; color de la envoltura de la semilla, beige claro; altura de la planta hasta la base de la inflorescencia, $77.8 \mathrm{~cm}$; forma del cariópside, ovoide; color, café oscuro; peso, 0.945 mg, largo, $1.79 \mathrm{~mm}$ y ancho de $1.11 \mathrm{~mm}$; inicio de imbibición, 3.0 h (0.125 días); imbibición completa, 8.0 h (0.33 días); emergencia de radícula, 10.0 h (0.42 días); emergencia del coleoptilo, 13.0 (0.54 días); velocidad de germinación, $5.77 \mathrm{pl} / \mathrm{h}$; días a emergencia, 7.00; vigor de la plántula, fuerte; capacidad de establecimiento, alto; mecanismo de rebrote, con yemas basales o de la corona radical y yemas axilares; grado de amacollamiento, excelente; vigor de la recuperación, excelente; días a emisión de flores, 65; tipo de floración, indeterminado; días al inicio de antesis, nueve; días a término de floración, indeterminado; número de semillas viables por inflorescencia, 20.

\section{Características agronómicas del buffel Titán}

La variedad Titán prospera bien donde la precipitación es igual o mayor a los $250 \mathrm{~mm}$ anuales, con buena distribución durante el verano y donde la temperatura media anual es de $16^{\circ} \mathrm{C}$, se desarrolla bien en suelos francos y franco arenosos. Es un pasto que ha registrado un buen crecimiento en altitudes de 1,800 a 1,950 m. Produce forraje de buena calidad, es tolerante a condiciones de aridez, protege al suelo contra la erosión y es resistente al pastoreo, es muy apetecido por el ganado. Presenta 
buena resistencia al acame: regular resistencia al desgrane, buena tolerancia a plagas y enfermedades, buena tolerancia a la sequía, excelente recuperación después de una quema, con regular tolerancia a la salinidad, regular tolerancia a acidez del suelo. No es tolerante a heladas por lo que en invierno permanece en latencia y al volver la primavera se recupera rápidamente. Presenta tolerancia regular a inundaciones $y$, en general, se considera que tiene buena persistencia.

De acuerdo a las evaluaciones realizadas durante el proceso de selección de la variedad, se estimó que el rendimiento medio en condiciones de temporal es de $2,120 \mathrm{~kg} \mathrm{MS} \mathrm{ha}^{-1}$. En un sitio con altitud de $1,882 \mathrm{~m}$, el rendimiento fue de 1,130, 2,090 y $2,370 \mathrm{~kg} \mathrm{ha}^{-1}$, cuando las precipitaciones fueron de $263.8,332.2$ y $368.5 \mathrm{~mm}$, con alturas de planta de 56,65 y $73 \mathrm{~cm}$, respectivamente. Con una altitud de 2,198 $\mathrm{m}$, los rendimientos han sido de $1,650,2,820,2,078$ y $2,010 \mathrm{~kg} \mathrm{ha}^{-1}$ con precipitaciones de 247.9, 420.2, 376.6 y $394.4 \mathrm{~mm}$ y alturas de planta de $51,71,65$ y $47 \mathrm{~cm}$.

En el lote de producción de semilla del Campo Experimental San Luis, de INIFAP, en condiciones de riego y fertilización (80-40-00), se han obtenido en promedio $527 \mathrm{~kg} \mathrm{ha}^{-1}$ de semilla con envoltura con porcentaje de germinación de 88.6 \% y pureza física de $92.4 \%$. La producción de forraje en condiciones de riego, ha variado de 5.18 a $8.24 \mathrm{MS} \mathrm{ha}^{-1}$.

\section{Características agronómicas del buffel Regio}

Buffel Regio es un material apto para las zonas áridas y semiáridas del altiplano Potosino, se desarrolla bien en suelos franco arenosos, donde la precipitación es igual o superior a $250 \mathrm{~mm}$ anuales, con buena distribución durante el verano y donde la temperatura media anual es de $16^{\circ} \mathrm{C}$. Produce excelente calidad de forraje, muy apetecible para el ganado, con buena resistencia al acame, regular resistencia al desgrane, con buena tolerancia a plagas y enfermedades, buena tolerancia a sequía, buena respuesta después de una quema, no es tolerante a heladas, con regular tolerancia a la salinidad, regular tolerancia a la acidez, presenta poca tolerancia a inundaciones y muestra buena persistencia. La producción de forraje en materia seca promedio es de $2,590 \mathrm{~kg} \mathrm{ha}^{-1}$, y la producción de semilla en lotes experimentales fue de $566.25 \mathrm{~kg}$ $\mathrm{ha}^{-1}$. Es un pasto que ha registrado buen desarrollo a altitudes de 1,800 a 2,100 m.

De acuerdo a las evaluaciones realizadas durante el proceso de selección de la variedad, se estimó que el rendimiento medio en condiciones de temporal es de $2,582 \mathrm{~kg} \mathrm{MS} \mathrm{ha}^{-1}$. El rendimiento a una altitud de $1,882 \mathrm{~m}$, fue de $1,828,2,620$ y 2,560 $\mathrm{kg} \mathrm{ha}^{-1}$, cuando las precipitaciones fueron de 263.8, 332.2 y $368.5 \mathrm{~mm}$, con alturas de planta de 62,69 y $78 \mathrm{~cm}$, respectivamente. En otro sitio con altitud de $2,198 \mathrm{~m}$, se obtuvieron rendimientos de $2,560 \mathrm{y}$ $2,970,2,390$ y $2,850 \mathrm{~kg} \mathrm{ha}^{-1}$ con precipitaciones de $247.9,420.2,376.6$ y $394.4 \mathrm{~mm}$ y alturas de planta de $62,69,75$ y $78 \mathrm{~cm}$.

En el lote de producción de semilla del Campo Experimental San Luis, de INIFAP, en condiciones de riego y fertilización (80-40-00), se obtuvo en promedio una producción de semilla con envoltura de $583 \mathrm{~kg} \mathrm{ha}^{-1}$, con porcentajes de germinación de $85.6 \%$ y una pureza de $93.6 \%$. La producción de forraje en condiciones de riego, ha variado de 6.35 a 9.16 t MS ha-1.

El valor nutricional de las variedades de buffel Titán y Regio es similar al reportado para esta especie $^{(18)}$. En el Cuadro 1 se muestran los resultados del análisis bromatológico de ambas variedades.

Cuadro 1. Valor nutricional de las variedades buffel Titán y buffel Regio en floración y madurez (\%)

\begin{tabular}{|c|c|c|c|c|c|c|c|c|c|c|c|}
\hline \multirow{2}{*}{ Variedad } & \multirow{2}{*}{$\begin{array}{c}\text { Materia seca } \\
(\%)\end{array}$} & \multicolumn{2}{|c|}{ PC } & \multicolumn{2}{|c|}{ PD } & \multicolumn{2}{|c|}{ Cenizas } & \multicolumn{2}{|c|}{ Calcio } & \multicolumn{2}{|c|}{ Fósforo } \\
\hline & & $\mathrm{F}$ & $M$ & $F$ & $M$ & $\mathrm{~F}$ & $\bar{M}$ & $F$ & $M$ & $F$ & $M$ \\
\hline Titán & 42 & 6.1 & 4.0 & 4.2 & 2.6 & 15.3 & 12.6 & 0.31 & 0.39 & 0.13 & 0.08 \\
\hline Regio & 39 & 5.8 & 3.3 & 2.9 & 1.9 & 14.3 & 12.0 & 0.32 & 0.43 & 0.09 & 0.03 \\
\hline
\end{tabular}

PC= Proteína cruda; $\mathrm{PD}=$ Proteína digestible; F= Floración; M= Madurez. 


\section{LITERATURA CITADA}

1. Hanselka CW, Hussey MA, Ibarra FFA. Buffelgrass. In: Moser LE, et al. Co-editors. Warm-Season (C4). GASA - CSSA - SSSA. Agronomy Monograph. Madison, Wi. USA. 2004;(45):477-502.

2. Ibarra FFA, Martin RMH, Hussey MA, Burson BL, Denogean BF, Moreno MS, Ayala AF. Variedades de zacate Buffel tolerantes al frío. INIFAP. Publicación Técnica No. 3. Campo Experimental Costa de Hermosillo. Sonora, México. 2011.

3. Ibarra FFA, Moreno MS, Martín RM, Denogean BF, Gerlach BLE. La siembra de zacate Buffel como una alternativa para incrementar la rentabilidad de los ranchos ganaderos de la sierra de Sonora. Tec Pecu Mex 2005;43(2):173-183.

4. Morales NCR. Características de los principales zacates forrajeros para zonas áridas. INIFAP. Folleto Técnico No. 2 CIRNOC. Campo Experimental La Campana. Chihuahua, México. 1994.

5. Beltrán LS, Loredo OC. Manejo y rehabilitación de agostaderos de zonas áridas y semiáridas. En: "Prácticas para la conservación del suelo y agua en zonas áridas y semiáridas" INIFAP-CIRNE-Campo Experimental San Luis. S.L.P. Libro técnico No. 1. 2005.

6. Loredo OC, Beltrán LS, Villanueva DJ, Urrutia MJ. Establecimiento de pasto Buffel para el control de la erosión hídrica. INIFAP-CIRNECampo Experimental San Luis. Folleto técnico No 26. 2005.

7. Beltrán LS, Loredo OC. Reconversión de áreas agrícolas marginales a praderas de pasto Buffel. INIFAP-CIRNE-Campo Experimental San Luis Potosí, S.L.P. Folleto técnico No. 36. 2002.

8. Gómez FE, Díaz SH, Saldívar FA, Briones EF, Vargas TV, Grant EW. Patrón de crecimiento del pasto buffel [Pennisetum ciliare L (Link). Sin. Cenchrus ciliaris L.] en Tamaulipas, México. Tec Pecu Mex 2007;45:1-17.

9. Celaya MH, García OF, Rodríguez JC, Castellanos VAE. Cambios en el almacenamiento de nitrógeno y agua en el suelo de un matorra desértico transformado a sabana de Buffel (Pennisetum ciliare (L.) Link). Terra Latinoamericana 2015;33:79-93.

10. Cruz MA, Pedroza SA, Trejo CR, Sánchez CI, Samaniego GJA, Cantú BJE. Alternativas y toma de decisiones en el manejo de los recursos naturales en áreas degradadas de zonas áridas. Revista Chapingo Serie Zonas Áridas. 2015;XIV:1:51-60.

11. Martínez LJR, Gutiérrez OE, Barrera SMA, Retes LR. Simulación estocástica para praderas de pasto Buffel (Cenchrus ciliaris L.) en Marín N. L. México. Trop Subtrop Agroecosystems 2014;17:87-104.

12. Burson BL, Actkinson JM, Hussey MA, Jessup RW. Ploidy determination of Buffelgrass accessions in the USDA National Plant Germoplasm System Collection by flow cytometry. S Afr Bot 2012;79:91-95.
13. Burson $B L$, Renganayaki $K$, Dowling $D C H$, Hinze $L L$, Jessup RW. Genetic diversity among pentaploid Buffelgrass accessions. Crop Sci 2015;55:1637-1645.

14. Beltrán LS, Hernández GA, Pérez PJ, García ME, Kohashi SJ, Herrera HJG. Respuesta fisiológica del pasto Buffel (Cenchrus ciliaris L.) a diferentes alturas de defoliación. Agrociencia 2002;36(5):547-556.

15. Beltrán LS, Hernández GA, García ME, Pérez PJ, Kohashi SJ, Herrera $\mathrm{HJG}$, et al. Efecto de la altura y frecuencia de corte en el crecimiento y rendimiento del pasto Buffel (Cenchrus ciliaris L.) en un invernadero. Agrociencia 2005;39:137-147.

16. Búrquez A. El zacate Buffel: Transformación ecológica y social. CONABIO. Biodiversitas 2007;74:8-12.

17. Martín HM, Ibarra FFA. Productividad y calidad forrajera. Guía práctica para el establecimiento, manejo y utilización del zacate Buffel (Cenchrus ciliaris L.). Publicación especial. Patrocipes. Hermosillo, Sonora. México. 1995.

18. Garcia DG, Ramírez LRG, Foroughbakhch R, Morales RR, García DG. Valor nutricional y digestión ruminal de cinco líneas apomíticas y un híbrido de pasto Buffel (Cenchrus ciliaris L.). Tec Pecu Mex 2003;41(2):209-218.

19. Hernández RP, Cuellar VEJ, Martínez VJ. Guía para el establecimiento y manejo de zacate Buffel Zaragoza 115 para la producción de semilla bajo riego. INIFAP-CIRNE-Campo Experimental Zaragoza. Coahuila, México. Folleto Técnico No. 13. 2004.

20. Velázquez VMA, Muñoz VJA, Macías RH, Esquivel AG, Rivera GM. Producción de forraje de variedades de zacate Buffel (Pennisetum ciliare L.) Link (Sin. Cenchrus ciliaris L.) en la región árida del estado de Durango. México. Agrofaz 2014;14(1):69-76.

21. Hussey MA, Burson BL. Registration of "Frio" Buffelgrass. Crop Sci 2005;45:411-412.

22. Silva OMF, Ramírez MF, Parra GMA, Martín RMH, Burboa CFR. Evaluación de 20 variedades de zacate Buffel (Cenchrus ciliaris L.) en el matorral arbosufrutescente de Sonora. Téc Pecu Méx 1993;31(1):51-55.

23. García DCA. Evaluación de gramíneas nativas e introducidas en el Altiplano Potosino. Tercera Reunión Científica. INIFAP-CIRNE-SLP. 1992.

24. Beltrán LS, Loredo OC, García DCA, Hernández AJA, Urrutia MJ, Gámez VHG, González ELA. Buffel Titán y Buffel Regio nuevas variedades de pastos para el Altiplano de San Luis Potosí. INIFAPCIRNE-Campo Experimental San Luis. Folleto Técnico No. 35. 2008. 